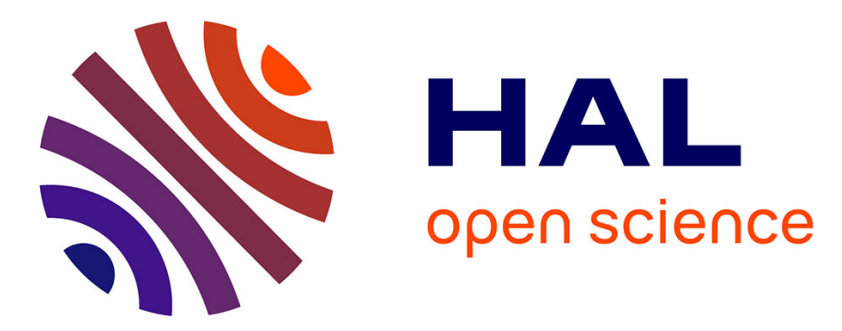

\title{
Assessment of a multi-reflectometers positioning system for DEMO plasmas
}

Emanuel Ricardo, Filipe da Silva, Stéphane Heuraux, Antonio Silva

\section{To cite this version:}

Emanuel Ricardo, Filipe da Silva, Stéphane Heuraux, Antonio Silva. Assessment of a multireflectometers positioning system for DEMO plasmas. Journal of Instrumentation, 2019, 14 (08), pp.C08010. 10.1088/1748-0221/14/08/C08010 . hal-02440048

\section{HAL Id: hal-02440048 \\ https://hal.univ-lorraine.fr/hal-02440048}

Submitted on 14 Jan 2020

HAL is a multi-disciplinary open access archive for the deposit and dissemination of scientific research documents, whether they are published or not. The documents may come from teaching and research institutions in France or abroad, or from public or private research centers.
L'archive ouverte pluridisciplinaire HAL, est destinée au dépôt et à la diffusion de documents scientifiques de niveau recherche, publiés ou non, émanant des établissements d'enseignement et de recherche français ou étrangers, des laboratoires publics ou privés. 


\title{
Assessment of a multi-reflectometers positioning system for DEMO plasmas
}

\author{
E. Ricardo, ${ }^{a}$ F. da Silva, ${ }^{a}$ S. Heuraux ${ }^{b}$ and A. Silva ${ }^{a}$ \\ ${ }^{a}$ Instituto de Plasmas e Fusão Nuclear, Instituto Superior Técnico, \\ Av. Rovisco Pais, $n^{\circ} 1$, Lisbon, Portugal \\ ${ }^{b}$ Institut Jean Lamour UMR 7198 CNRS-Université de Lorraine, \\ ARTEM BP 50840, F-54011 Nancy, France \\ E-mail: ericardo@ipfn.tecnico.ulisboa.pt
}

Aвstract: Microwave reflectometry is the prime candidate to measure the DEMO plasma position and shape. DEMO will be based on a pre-defined operation scenario, allowing the optimization of the reflectometry measurements according to the expected parameters. When evaluating the system performance for the expected baseline scenario, it is essential to take into account the effects of the plasma displacement, of the turbulence and of the MHD activity in the measurements. Therefore, the design of the DEMO plasma position reflectometer (DEMO PPR) involves the assessment of the measurement performance of different poloidal views, antenna assemblies, emitting angles and different plasma configurations. In this work we evaluate the measurement performance over 100 different poloidal positions of the DEMO PPR using a synthetic monostatic O-mode reflectometer. The antennas were initially aligned perpendicularly to the wall. The results show that most of the locations at the top of the machine and near the divertor are not adequate for measuring the plasma position. These locations are characterized by larger wall-plasma distances and by larger angle of incidence on the separatrix. In order to optimize the measurement performance of the system, the antennas were aligned perpendicularly to the expected separatrix position, reducing these parameters. With the exception of the locations near the divertor, this geometrical consideration improved the measurement performance for all the positions, placing them below the requirement for the plasma positioning $(\sim 1 \mathrm{~cm})$. The locations near the divertor lose the signal due to the significant plasma curvature before the separatrix. We also studied the effect of a vertical plasma displacement of $\Delta z= \pm 5 \mathrm{~cm}$ in the measurements. The results show that the system is stable, fulfilling the chosen requirements. Aligning the emission perpendicularly to the separatrix proved to be a necessary condition in the optimization and stability of the DEMO PPR system.

KEYwORDs: Nuclear instruments and methods for hot plasma diagnostics; Microwave Antennas

This is an author-created, un-copyedited version of an article accepted for publication/published in Journal of Instrumentation. IOP Publishing Ltd is not responsible for any errors or omissions in this version of the manuscript or any version derived from it. The Version of Record is available online at 10.1088/1748-0221/14/08/C08010. 


\section{Contents}

1 Introduction 1

2 Review of the input models and measured quantities 2

3 The measurement performance of the multi-reflectometers system 4

4 Conclusion and future work $\quad 6$

\section{Introduction}

DEMO will be the first electrical power generating fusion machine. Unlike the experimental tokamaks, DEMO will follow a specific operating scenario and the diagnostic system will be set to the essential for machine protection and plasma control [1]. One of the fundamental measurements for plasma control is the plasma position and shape. In the present devices, it is measured with the magnetic diagnostics. However, this diagnostic faces two problems when considering its use in the DEMO and in other future machines. Firstly, large integrator drifts can occur during the long plasma pulses. Secondly, induced voltages can appear in the magnetic coils due to the high levels of radiation [2]. This can lead to a wrong plasma position measurement, putting operation at risk. In order to solve this problem in ITER, an O-mode reflectometer using 4 poloidal views was proposed as a supplementary diagnostic to backup and complement the magnetic-based control [3]. Microwave reflectometry is a diagnostic capable of measuring electron density profiles in the edge [4]. If the separatrix density is known, one can measure its position. The O-mode measurements are independent from the magnetic field, making this mode suitable for replacing the magnetic diagnostics. This technique was recently verified and validated in the ASDEX-Upgrade [5]. Microwave diagnostics, like reflectometry or electron cyclotron emission, are the preferred diagnostics for DEMO, fulfilling the diagnostic requirements in the context of a fusion reactor: the reduced access, the robustness, the reliability and the resolution. For this reason, microwave reflectometry is the prime candidate to measure the entire DEMO plasma position and shape [1,2]. The DEMO plasma position reflectometer (DEMO PPR) will be a system of multi-reflectometers distributed over the wall with the purpose of measuring the position and shape of the plasma.

The position error of a reflectometer depends on its geometry, on the plasma shape and on the plasma-wall multiple-reflections. In the future fusion machines like DEMO, the reflectometers can be designed and optimized according to the expected plasma parameters and wall geometry. The plasma displacement, the turbulence and the MHD activity are important phenomena that occur during the discharge and must be taken into account when evaluating the system performance. Therefore, the design of the DEMO PPR involves the assessment of the measurement performance of different poloidal views, antenna assemblies, emitting angles and different plasma configurations. The DEMO PPR is in an early development stage and there are many ideas that must be discussed to 
reach the final design of the system [6]. As a first problem, it is fundamental to investigate the regions of the machine that are compatible with the measurement constraints for position control. In this work we evaluate the measurement performance of 100 different DEMO poloidal positions using REFMULF, a FDTD code for reflectometry simulations [7]. Two different emission configurations were considered. In the first one the antennas were aligned perpendicularly to the wall and in the second they were aligned perpendicularly to the separatrix. We also evaluated the effect of a plasma vertical displacement of $\Delta z \pm 5 \mathrm{~cm}$ in the second configuration.

\section{Review of the input models and measured quantities}

From the EUROFUSION official database we have (i) the vacuum vessel geometry, (ii) the poloidal flux, (iii) the separatrix line and (iv) the electron density profile for the 2015 baseline scenario. The data presents two limitations: (i) the lack of information in the scrape off layer region and (ii) the poor resolution of the $2 \mathrm{D}$ poloidal flux data comparing with the FDTD simulation grid. To overcome the first problem, we extrapolated the density profile linearly, as shown in the figure 1 (left).
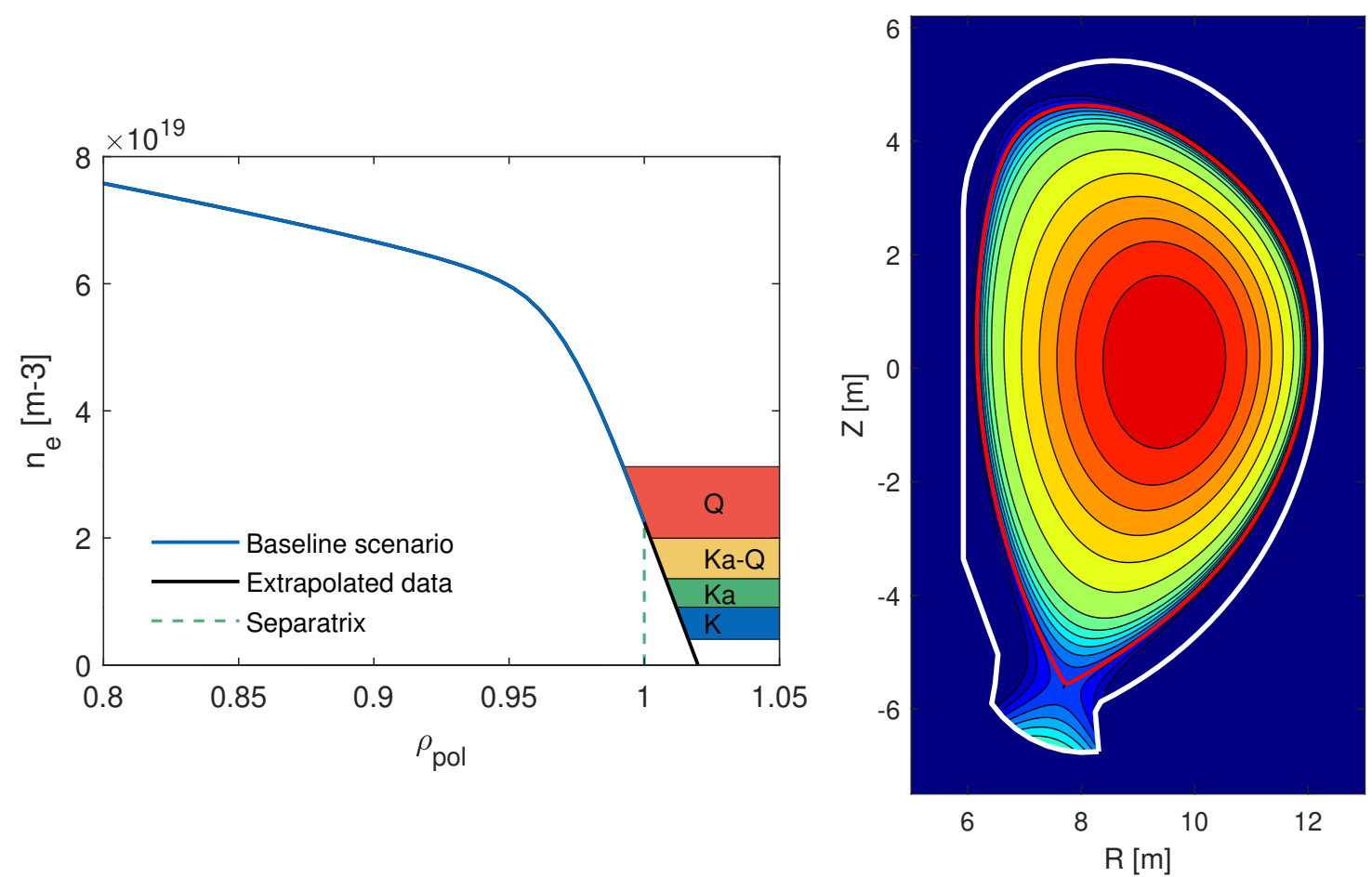

Figure 1. The DEMO baseline scenario electron density with the linear extrapolation in the scrape-off layer region (left) and the obtained density map $n_{e}(R, Z)$ (right).

By sweeping the frequency and measuring the round trip time delay, the position of a layer is calculated using the Abel Inversion [4]. Each band probes the plasma at different zones according to the O-mode cut-off density expression, $n_{c}(F)=\alpha F^{2} . \quad F$ is the probing frequency and $\alpha \simeq$ $80.6545 \mathrm{~m}^{-3} \mathrm{~s}^{2}$. Thus, we use the K-Ka-Q bands $(18-50 \mathrm{GHz})$ to probe the DEMO separatrix. Having $\rho_{\mathrm{pol}}(R, Z)$ and $n_{e}\left(\rho_{\mathrm{pol}}\right)$, we obtained the density map $n_{e}(R, Z)$ shown in figure 1 (right). The second problem was solved by interpolating the density map to the FDTD grid dimensions. 
A recent study indicates that at least 15 measurements are required for a reliable reconstruction of the DEMO separatrix [8]. The maximum number of measurements is defined by the spatial constrains imposed to the waveguides. With the aim of studying the performance of the different regions of the machine, we defined 100 positions around the wall, in agreement with the concept of slim cassette [9]. The positions, known as gaps, were divided in 16 regions and labeled by the code G[region][letter]. For this system of positions, we defined two different emission configurations, one with the emission line aligned perpendicularly to the wall and the other with the emission line aligned perpendicularly to the separatrix. For each position and configuration, we defined the boxes to place the antenna assembly models and the respective regions of interest (ROI) for the simulations. Figure 2 shows the representation of antenna assembly boxes for the two configurations. Two regions of interest are represented, corresponding to the gaps G6 and G13.
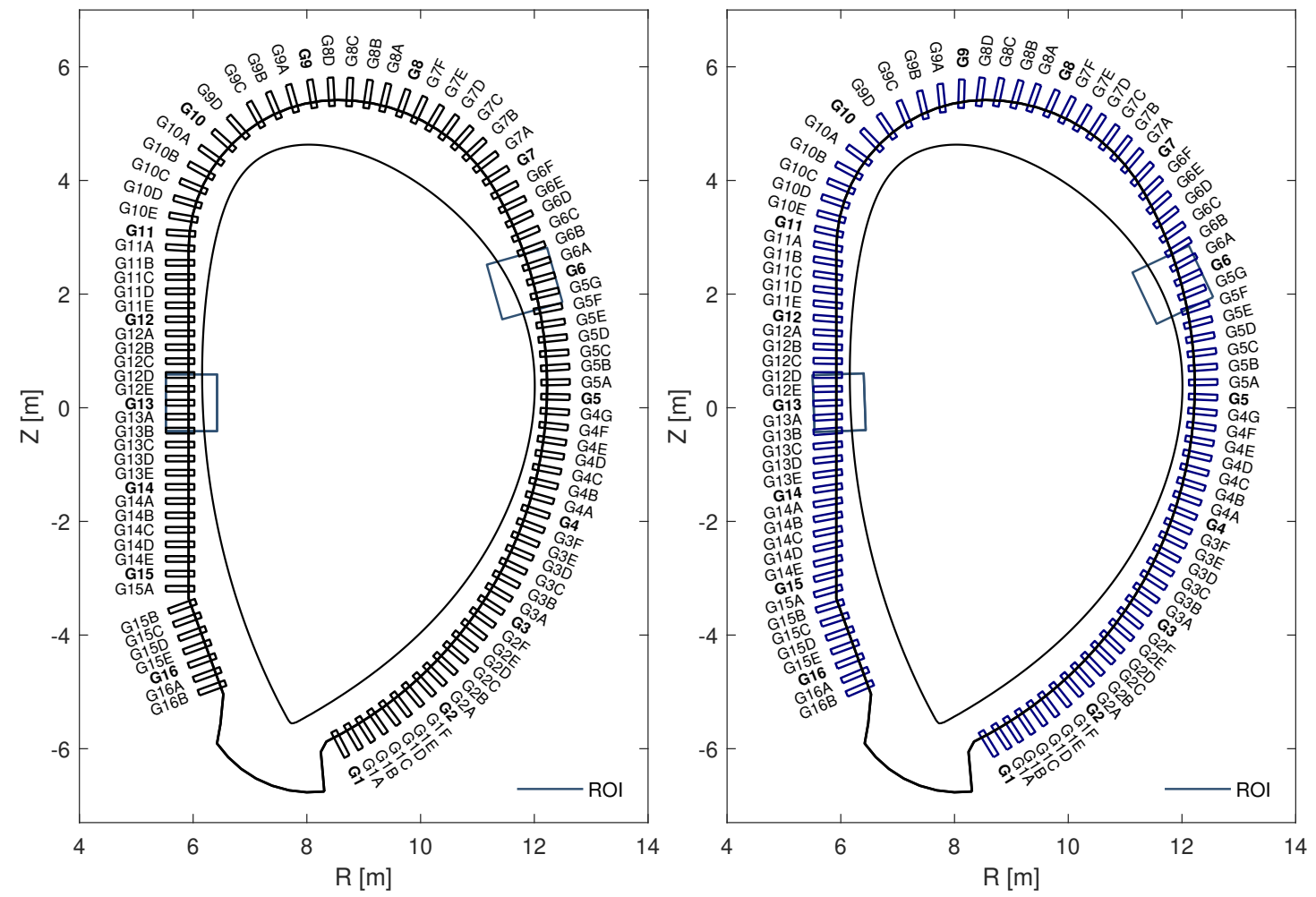

Figure 2. The first configuration with the emission line perpendicular to the wall (left) and second configuration with the emission line perpendicular to the separatrix (right).

The plasma position error is given by the expression

$$
\operatorname{Error}(F)=\left|r(F)-r_{0}(F)\right|=\left|\frac{c}{2 \pi^{2}} \int_{0}^{F}\left(\frac{\partial \varphi}{\partial f}-\frac{\partial \varphi_{0}}{\partial f}\right) \frac{d f}{\sqrt{F^{2}-f^{2}}}\right|
$$

$r(F)$ and $\varphi$ are, respectively, the position and the phase calculated with the simulation results. $r_{0}(F)$ and $\varphi_{0}$ are the position and the phase calculated with the WKB expression [4]. We apply the IQ detection method with an implemented 5th order butterworth filter to obtain the phase derivative from the simulations. The detection is performed in a fundamental waveguide localized before a taper connected to the antenna's oversized waveguide. This allows detecting only the fundamental 
mode, as in the real experiments. The signal is initialized in the $0-18 \mathrm{GHz}$ range with the WKB phase derivative. The measurement performance of a multi-reflectometers positioning system is the separatrix position error as a function of the gap, $\operatorname{Error}\left(F=f_{\text {sep }}\right.$, gap). It is assumed a requirement of $1 \mathrm{~cm}$ for the plasma positioning system, similarly to the ITER PPR [3].

\section{The measurement performance of the multi-reflectometers system}

Figure 3 shows the measurement performance of the PPR for the first emission configuration and for the baseline scenario. Only the first gaps of each region G1-16 are represented in the Gap axis.
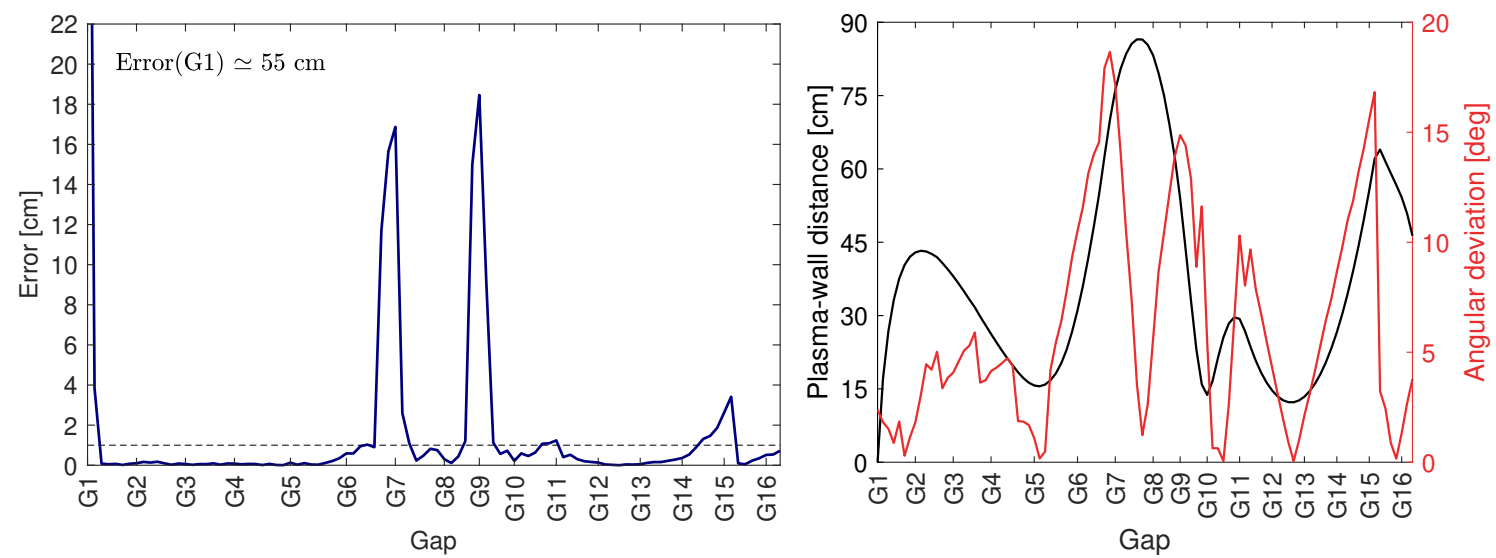

Figure 3. The measurement performance of the PPR for the first configuration (left). The wall-plasma distance and the angular deviation (angle of incidence on the separatrix) (right).

The results show that the positions in the equatorial plane region and some below (G1C-G6 and G11C-G14) exhibit errors in the order of $1 \mathrm{~mm}$, being in agreement with the requirements for plasma positioning. The positions near the divertor (G1A-B and G14B-G15A) and at the top of the machine (G6B-G7B, G8C-G9B and G10D-G11) have errors in the order or greater than $1 \mathrm{~cm}$. They correspond to the gaps where the angle between the emission and the line perpendicular to the separatrix (the angular deviation) and the distance between the wall and the initial plasma position (the plasma-wall distance) are larger simultaneously. Both are shown in figure 3. The peaks correspond to the limiting cases where the signal is lost due to the significant plasma curvature. The figure 4 (left) shows a snapshot of the electric field of the gap G7 at an emission frequency of $46 \mathrm{GHz}$ where this effect is represented. The figure 4 (right) shows the electric field structure for the gap G12D, corresponding to the result of the multireflection between the cut-off and the wall. The density lines are approximately perpendicular to the emission line. The reflectometry mathematical model assumes a plane wave propagating perpendicularly to a one dimensional varying slab plasma, explaining why the gaps with approximately these conditions have the lower separatrix position errors. In order to optimize the measurement performance of all the system, a second configuration of the antennas with the emission aligned perpendicularly to the separatrix was defined (figure 2).

With this geometrical assumption the wall-plasma distance and the emission angular deviation are minimized. When the beam is reflected from the plasma, the plasma curvature can induce a 

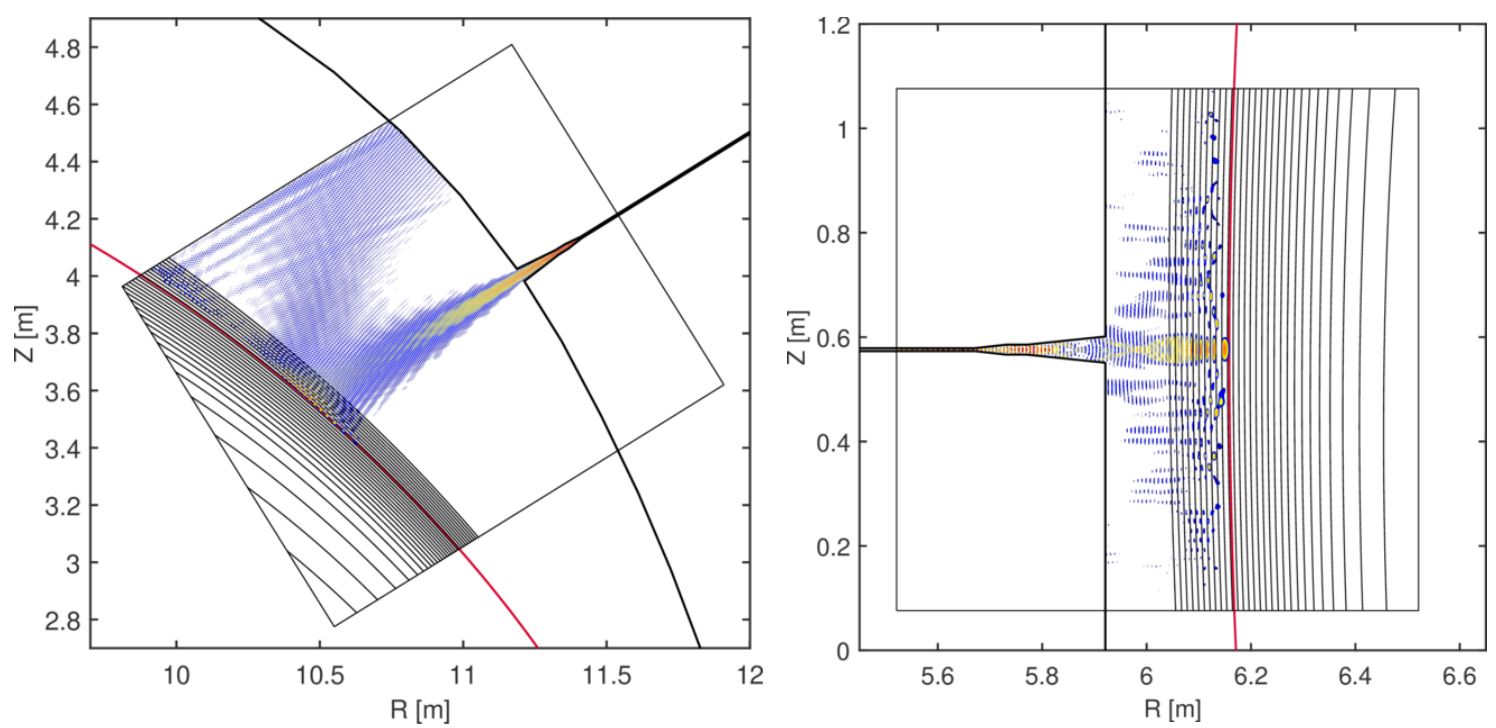

Figure 4. The electric field snapshot for the gap G7, at $46 \mathrm{GHz}$ (left) and the electric field snapshot for the gap G12D at $46 \mathrm{GHz}$ (right).

propagation direction different from the initial one, reducing the detected amplitude and contributing to obtain a wrong phase variation. Reducing the wall-plasma distance, is expected optimizing both effects. By reducing the angular deviation to zero, the emission is perpendicular to the separatrix. This condition is not enough to guarantee a perpendicular emission with respect to the other layers of the frequency range. However, in most of the regions in the machine the flux surfaces are locally approximately parallel. Besides that, the measurement of the position of a layer depends strongly on the region near the cut-off, so we expect a lower contribution of the lower frequency layers. Figure 5 shows the measurement performance for the second configuration (left).
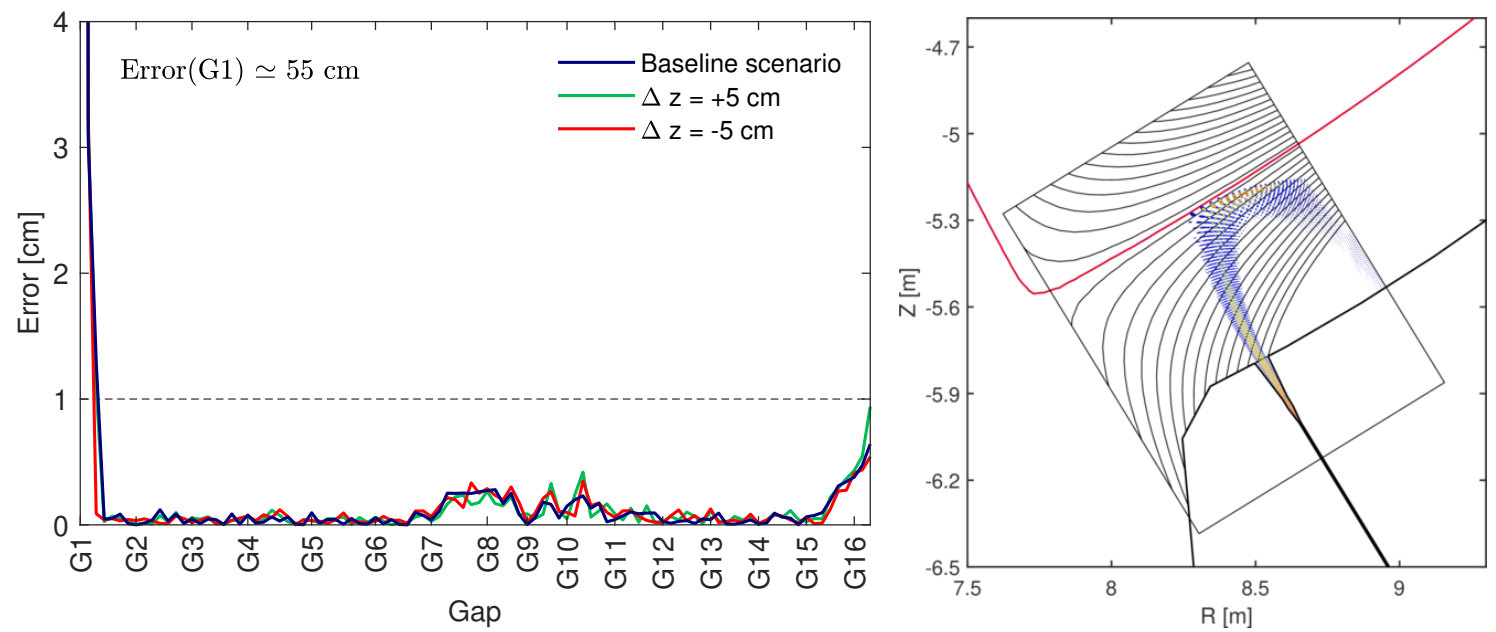

Figure 5. The measurement performance of the second configuration and the effects of a vertical displacement of $\Delta z= \pm 5 \mathrm{~cm}$ (left) and the electric field snapshot of the gap G1 at $46 \mathrm{GHz}$ (right). 
As expected, most of the gaps improved the performance. For all the positions around the machine the error is below the requirement of $1 \mathrm{~cm}$, with the exception of the divertor region. For these positions, the plasma has a large poloidal density gradient which requires a specific procedure to improve the separatrix position measurements, so an ad hoc procedure should be developed. In the limiting cases, the beam is totally deviated. This occurs for the gap G1, as shown in the figure 5 (right). If a measurement at these locations is essential to achieve a reliable plasma shape reconstruction, an optimization of the emission angle has to be done and/or a solution with multiple emitting and/or receiving antennas has to be adopted.

The plasma displacements can increase the wall-plasma distance and the emission angular deviation leading to wrong position measurements. For this reason, we assessed the effects of having a vertical displacement of $\Delta z= \pm 5 \mathrm{~cm}$, the magnitude assumed in the reference [10]. The results are shown in the figure 5 (left). The system maintains its measurement performance for vertical displacements of this order.

\section{Conclusion and future work}

Microwave reflectometry is the prime candidate to measure the DEMO plasma position and shape. The DEMO plasma position reflectometer (PPR) can be designed and optimized by evaluating the measurement performance of different poloidal views, antenna assemblies, emitting angles and different plasma configurations. One of the fundamental questions of this early development stage is which are the regions of the machine that allow to have measurements within the limiting requirements for position control and if it is possible to optimize them.

In this work we used a synthetic monostatic reflectometer to assess the measurement performance of 100 positions distributed along the tokamak's wall. In the first study the emission was aligned with the wall, and the system exhibited unacceptable measurement errors, outside DEMO requirements, for the regions at the top of the machine and near the divertor region. With the aim of optimizing the system performance, the gaps were aligned perpendicularly to the separatrix. The results confirmed this principle. By aligning the emission perpendicularly with the separatrix, the measurement performance of the system improved for all the positions, with the exception of the divertor. In these locations, the plasma curvature is so strong that deviates the beam before it reaches the separatrix layer. If it's essential to have a measurement at this location, a study of the best emitting angles to measure the plasma position must be performed and a system of multiple receiving and emitting antennas can be considered. Another important question of a reflectometry system is its stability under the vertical displacements of the plasma. The system proved to be stable for vertical displacements of $\Delta z= \pm 5 \mathrm{~cm}$ of the DEMO baseline scenario. One interesting future study is to use different angles and magnitudes of displacement to understand the system limits.

Aligning the emission perpendicularly to the separatrix proved to be a fundamental geometrical assumption in the optimization and stability of the measurement performance of the DEMO PPR. Being the system optimized for the baseline scenario and its stability tested for different vertical displacements, it's essential to study the effects of the turbulence and MHD activity in the measurement performance of the DEMO multi-reflectometers positioning system. 


\section{Acknowledgments}

This work has been carried out within the framework of the French Federation for Magnetic Fusion Studies (FR-FCM) and of the EUROfusion consortium, and has received funding from the Euratom research and training programme 2014-2018 and 2019-2020 under grant agreement No 633053. The views and opinions expressed herein do not necessarily reflect those of the European Commission. IST activities also received financial support from Fundação para a Ciência e Tecnologia through project UID/FIS/50010/2019. Part of the simulations were performed at the IST cluster (Lisbon, Portugal).

\section{References}

[1] W. Biel, M. de Baar, A. Dinklage, F. Felici, R. König, H. Meister et al., DEMO diagnostics and burn control, Fusion Eng. Des. 96-97 (2015) 8.

[2] W. Biel et al., Diagnostics for plasma control - From ITER to DEMO, Fusion Eng. Des. (2019), in press.

[3] F. da Silva, S. Heuraux, E. Ricardo, P. Quental and J. Ferreira, Assessment of the measurement performance of the in-vessel system of gap 6 of the ITER plasma position reflectometer using a finite-difference time-domain maxwell full-wave code, Rev. Sci. Instrum. 87 (2016) 11E727.

[4] E. Mazzucato, Microwave reflectometry for magnetically confined plasmas, Rev. Sci. Instrum. 69 (1998) 2201.

[5] J. Santos, L. Guimarãis, M. Zilker, W. Treutterer and M. M. and, Reflectometry-based plasma position feedback control demonstration at ASDEX upgrade, Nucl. Fusion 52 (2012) 032003.

[6] A.G. Silva, F. da Silva, S. Heuraux and B. Gonçalves, First assessment of microwave diagnostics for DEMO, Fusion Eng. Des. 96-97 (2015) 948.

[7] F. da Silva, S. Heuraux, S. Hacquin and M. Manso, Unidirectional transparent signal injection in finite-difference time-domain electromagnetic codes-application to reflectometry simulations, $J$. Comp. Phys. 203 (2005) 467.

[8] G. Marchiori, G. D. Masi, R. Cavazzana, A. Cenedese, N. Marconato, R. Moutinho et al., Study of a plasma boundary reconstruction method based on reflectometric measurements for control purposes, IEEE Trans. Plasma Sci. 46 (2018) 1285.

[9] A. Malaquias, A. Silva, R. Moutinho, R. Luis, A. Lopes, P. B. Quental et al., Integration concept of the reflectometry diagnostic for the main plasma in DEMO, IEEE Trans. Plasma Sci. 46 (2018) 451.

[10] R. Wenninger, F. Arbeiter, J. Aubert, L. Aho-Mantila, R. Albanese, R. Ambrosino et al., Advances in the physics basis for the european DEMO design, Nucl. Fusion 55 (2015) 063003. 